\title{
Incidence of delayed and missed diagnoses in whole-body multidetector CT in patients with multiple injuries after trauma
}

\author{
Lucas L Geyer ${ }^{1}$, Markus Körner' ${ }^{1}$, Ulrich Linsenmaier ${ }^{1}$, Stefan Huber-Wagner², \\ Karl-Georg Kanz ${ }^{3}$, Maximilian F Reiser ${ }^{1}$ and Stefan Wirth' \\ ${ }^{1}$ Department of Clinical Radiology, University Hospitals LMU Munich; ${ }^{2}$ Department of Trauma Surgery, Klinikum rechts der Isar, Technical \\ University of Munich; ${ }^{3}$ Department of Surgery, University Hospitals LMU Munich, Germany \\ Correspondence to: Lucas L Geyer. Email: lucas.geyer@med.Imu.de
}

\begin{abstract}
Background: Whole-body CT (WBCT) is the imaging modality of choice during the initial diagnostic work-up of multiple injured patients in order to identify serious injuries and initiate adequate treatment immediately. However, delayed diagnosed or even missed injuries have been reported frequently ranging from $1.3 \%$ to $47 \%$.

Purpose: To highlight commonly missed lesions in WBCT of patients with multiple injuries.

Material and Methods: A total of 375 patients (age $42.8 \pm 17.9$ years, ISS $26.6 \pm 17.0$ ) with a WBCT (head to symphysis) were included. The final CT report was compared with clinical and operation reports. Discrepant findings were recorded and grouped as relevant and non-relevant to further treatment. In both groups, an experienced trauma radiologist read the CT images retrospectively, whether these lesions were missed or truly not detectable.

Results: In 336 patients (89.6\%), all injuries in the regions examined were diagnosed correctly in the final reports of the initial CT. Forty-eight patients $(12.8 \%)$ had injuries in regions of the body that were not included in the CT. Fourteen patients $(3.7 \%)$ had injuries that did not require further treatment. Twenty-five patients $(6.7 \%)$ had injuries that required further treatment. With secondary interpretation, $85.4 \%$ of all missed lesions could be diagnosed in retrospect from the primary CT data-set. Small pancreatic and bowel contusions were identified as truly non-detectable.

Conclusion: In multiple traumas, only a few missed injuries in initial WBCT reading are clinically relevant. However, as the vast majority of these injuries are detectable, the radiologist has to be alert for commonly missed findings to avoid a delayed diagnosis.
\end{abstract}

Keywords: MDCT, multiple trauma, whole-body CT, missed injuries, delayed diagnosis

Submitted June 20, 2012; accepted for publication December 12, 2012

The main challenge for departments dealing with trauma patients who have multiple injuries is to diagnose those injuries that are severe and life-threatening and to initiate targeted and adequate treatment without delay. Multidetector CT (MDCT) is an excellent tool for imaging these patients, and its diagnostic accuracy and benefits over different imaging methods have been described extensively $(1-11)$.

Therefore, CT is an integrated part of the primary algorithm for resuscitation after trauma in many trauma centers, and is used routinely in patients with suspected multiple injuries $(2,12)$. A standard whole-body examination including head, chest, abdomen, and pelvis is done routinely after initial physical examination and on the indication given by the trauma surgeons and radiologists.

However, injuries that are diagnosed with delay, or even missed, are commonly reported in patients with multiple injuries and have been reported to range from $1.3 \%$ to $47 \%$ (13-22). These studies were, however, heterogeneous in terms of definition of delayed diagnoses or missed lesions, imaging methods, and clinical standards used, and did not focus mainly on the role of radiology or CT.

The purpose of this study was to evaluate the incidence and types of lesions that were not reported by the primary whole-body CT (WBCT) scan in patients with multiple injuries. 
Table 1 Imaging parameters for the standard WBCT

\begin{tabular}{llll}
\hline & Head & Chest & Abdomen \\
\hline Tube voltage (kV) & 120 & 120 & 120 \\
Effective mAs & 210 & $140^{*}$ & $200^{*}$ \\
Collimation $(\mathrm{mm})$ & $4 \times 1^{\dagger}$ & $4 \times 2.5$ & $4 \times 2.5$ \\
Slice thickness $(\mathrm{mm})$ (kernel) & 2 and 5 (brain) & 5 (soft tissue) & 5 (soft tissue) \\
& 2 (bone) & 3 (bone) & 3 (bone) \\
Table movement (mm) & 4 & 12.5 & 12.5 \\
Contrast $(\mathrm{mL})$ & $\varnothing$ & $120(300 \mathrm{mg} / \mathrm{mL}$ iodine) & 3 (bone) \\
Scan delay $(\mathrm{s})$ & $\varnothing$ & $3 \mathrm{~mL} / \mathrm{s}$ injection rate & 80 \\
\hline
\end{tabular}

*Dose modulation

tSequential scan mode

MPR, multiplanar reformations

\section{Material and Methods}

\section{Patients}

The institutional review board approved the retrospective analysis of patient records, and a waiver of consent was granted. Over a period of 3 years (from January 2003 to December 2005), records of all trauma patients who had a standardized four-detector row WBCT (head, chest, abdomen, pelvis, including the complete spine; Table 1) after admission to our level I trauma center were reviewed. Patients who did not have complete data available (e.g. missing CT report, discharge report, immediate death without further diagnostic proof) or did not have a CT according to the study protocol were excluded. The demographic and clinical data (including age, gender, mechanism of trauma, surgical and discharge reports) as well as the Injury Severity Score (ISS) were recorded.

\section{Analysis of data}

The final written reports of the initial CT examinations were retrieved and further analyzed for the purpose of this study. In clinical routine, all studies were read immediately following the CT examination by a senior resident or fellow on call with at least 3 years of experience. The radiologist on call had a 20 -h shift on weekdays (12:00 to 08:00) and 24-h shift on weekends (08:00 to 08:00). In terms of quality assurance, all initial reports were ultimately read and crosschecked by an attending radiologist, defined as final written report. The time interval between the initial reading by the radiologist in charge and the cross-check by the attending was variable depending on the time at which the CT scan was performed. During regular working hours (i.e. 08:00 to 17:00) the attending radiologist was present at the department and cross-checked the initial report immediately. During off-hours, all images and CT reports were reviewed at the beginning of the following working day. However, the analysis of discrepancies between the initial report and the clinical secondary reading was not part of this study.

All radiological diagnoses of the final written report were compared with the corresponding clinical records including reports from surgery, interventions, and final discharge diagnoses from the hospital information system. This combination of clinical sources was chosen as the clinical reference standard because of the fact that each of these components has specific diagnostic values as well as limitations. In daily patient care, there is no additional tool for evaluation, but the summary of the mentioned tests and procedures.

In all patients with discrepant findings, the specific CT examinations were re-evaluated by a fellow radiologist specialized in trauma and emergency radiology (with 6 years of experience). The radiologist was aware of the missed findings and was instructed to specifically analyze those injuries. The intention of this secondary reading was to evaluate whether the injuries were present in the CT data-set and could have been diagnosed, or if they could not be diagnosed from the data-set for other reasons (technical or methodical limitations) even if the definitive diagnose was known. It was not the purpose of this secondary evaluation to analyze the performance of the initial readers.

\section{Missed injuries}

Missed injuries were defined as lesions that were related to the trauma and present on admission, but not described in the report of the initial whole-body CT examination. Only those injuries that were located in the examination volume from head to symphysis were included; other injuries (such as to the extremities) were recorded but not rated as missed if they were not mentioned in the reports. According to the findings, the patients were divided into three groups: (i) no missed injuries; (ii) missed injuries of no further relevance; and (iii) missed injuries of clinical relevance. This relevance was defined by a need for specific treatment of a lesion or if a lesion was considered as an indicator of a severe injury.

\section{Statistical analysis}

The proportion of missed lesions was calculated for each group. The ISS for each group was also separately calculated and compared with the Mann-Whitney U test. Statistical software (SPSS 14.0, SPSS Inc., Chicago, IL, USA) was used for the calculations.

\section{Results}

From January 2003 to December 2005, a total of 581 trauma patients with multiple injuries were admitted. A total of 206 
Table 2 Distribution of injuries according to CT findings and clinical records

\begin{tabular}{|c|c|c|}
\hline Body region & Subcategory & $\begin{array}{l}\text { Patients, } \\
n(\%)\end{array}$ \\
\hline \multirow[t]{5}{*}{ Head/Neck } & & $135(36.0)$ \\
\hline & Intracranial bleedings & $81(21.6)$ \\
\hline & Midface & 71 (18.9) \\
\hline & Skull (including skull base) & $64(17.1)$ \\
\hline & Larynx & $3(0.8)$ \\
\hline \multirow[t]{7}{*}{ Abdomen } & & $51(13.6)$ \\
\hline & Spleen & $23(6.1)$ \\
\hline & Liver & $18(4.8)$ \\
\hline & Small/large bowel/mesentery & $16(4.3)$ \\
\hline & Kidneys & $10(2.7)$ \\
\hline & Pancreas & $4(1.1)$ \\
\hline & Stomach & $1(0.3)$ \\
\hline \multirow[t]{9}{*}{ Thorax } & & $143(38.1)$ \\
\hline & Rib cage & $91(24.3)$ \\
\hline & Pleural space (pneumo-, hemothorax) & $71(18.9)$ \\
\hline & Lung & $63(16.8)$ \\
\hline & Scapula & $29(7.7)$ \\
\hline & Clavicle & $21(5.6)$ \\
\hline & Sternum & $13(3.5)$ \\
\hline & $\begin{array}{l}\text { Mediastinum (hematoma, } \\
\text { pneumomediastinum) }\end{array}$ & $13(3.5)$ \\
\hline & Diaphragm & $1(0.3)$ \\
\hline \multirow[t]{4}{*}{ Pelvis } & & $43(11.5)$ \\
\hline & Pelvic girdle & $34(9.1)$ \\
\hline & Retroperitoneal hematoma & $23(6.1)$ \\
\hline & Genitourinary system & $8(2.1)$ \\
\hline \multirow[t]{4}{*}{ Spine } & & $95(25.3)$ \\
\hline & Lumbar spine & $52(13.9)$ \\
\hline & Thoracic spine & $36(9.6)$ \\
\hline & Cervical spine & $27(7.2)$ \\
\hline \multirow{5}{*}{$\begin{array}{l}\text { Upper } \\
\text { extremity }\end{array}$} & & \\
\hline & & $71(18.9)$ \\
\hline & Lower arm & $42(11.2)$ \\
\hline & Upper arm & $25(6.7)$ \\
\hline & Hand/wrist & $17(4.5)$ \\
\hline \multirow{5}{*}{$\begin{array}{l}\text { Lower } \\
\quad \text { extremity }\end{array}$} & & \\
\hline & & $97(25.9)$ \\
\hline & Lower leg & $49(13.1)$ \\
\hline & Upper leg & $47(12.5)$ \\
\hline & Foot/ankle & $28(7.5)$ \\
\hline \multirow[t]{4}{*}{ Vessels } & & $29(7.7)$ \\
\hline & Arterial injury & $24(6.4)$ \\
\hline & Aortic injury & $6(1.6)$ \\
\hline & Venous injury & $6(1.6)$ \\
\hline
\end{tabular}

patients were excluded because they did not have a WBCT $(n=69)$ or documentation was incomplete $(n=137)$. Of the remaining 375 patients, $289(77.1 \%)$ were men and 86 $(22.9 \%)$ were women. The mean ISS was $25.8( \pm 17.0 \mathrm{SD})$, and mean age was 42.8 years ( \pm 17.9 years SD). The vast majority of injuries were caused by blunt trauma (96\%). The causes of trauma were distributed as follows: motor vehicle accident $56 \%$, fall from height $21 \%$, and $23 \%$ due to other mechanisms of trauma (e.g. suicide, assault). A detailed analysis of the frequency of injuries is displayed in Table 2.

In 336 patients $(89.6 \%)$, all injuries in the included regions were primarily diagnosed correctly and included in the final reports of the initial CT. Fourteen patients $(3.7 \%)$ had 14 primarily missed injuries that did not require further treatment. Twenty-five patients $(6.7 \%)$ had 34 missed injuries that were relevant to the further clinical course. Missed
Table 3 Missed injuries in the initial CT report with no further clinical relevance

\begin{tabular}{lc}
\hline Type of injury & $\boldsymbol{n}$ \\
\hline Rib fractures & 5 \\
Lateral vertebral process fracture (lumbar spine) & 4 \\
Nasal bone fracture & 2 \\
Scapula fracture & 1 \\
Zygoma fracture & 1 \\
Spinous process fracture (cervical spine) & 1 \\
Total & 14 \\
\hline
\end{tabular}

Table 4 Missed injuries in the initial CT report that needed or initiated further treatment

\begin{tabular}{ll}
\hline Type of injury & $\boldsymbol{n}$ \\
\hline Colon serosal tear & 3 \\
Intraperitoneal bladder rupture & 2 \\
Shoulder fracture & 2 \\
Sacrum fracture & 2 \\
Contusion of colon & 2 \\
Epidural hematoma & 1 \\
Diffuse axonal injury & 1 \\
Bleeding from internal iliac artery & 1 \\
Rupture of iliac vein & 1 \\
Pneumatocele & 1 \\
Pneumopericardium & 1 \\
Small bowel contusion & 1 \\
Hemopneumothorax & 1 \\
Lung contusion & 1 \\
Fracture of mandibular collum & 1 \\
Fracture of occipital condyle & 1 \\
Serial rib fracture & 1 \\
Symphysis fracture & 1 \\
Kidney infarction & 1 \\
Contusion of pancreas & 1 \\
Fracture of pubic arch & 1 \\
Pneumothorax & 1 \\
Fracture of sternum & 1 \\
Duodenal rupture & 1 \\
Stable lumbar vertebral body fracture & 1 \\
Pneumomediastinum & 1 \\
Hemotympanon & 1 \\
Intracranial air collection & 1 \\
Total & 1 \\
\hline
\end{tabular}

injuries are shown in Tables 3 (clinically not relevant) and 4 (clinically relevant). In addition, 48 patients $(12.8 \%)$ had injuries in body regions that were not included in the CT.

The initial CT report did not mention any injury in $85(22.7 \%)$ patients: four cases were considered as falsenegative (each CT report missed a single injury: two fractures of the sacrum, one fracture of the mandible, one fracture of a lumbar transverse process); 22 cases had osseous injuries of the extremities which were outside of the CT scan volume, but were diagnosed by additional plain radiography; 59 cases had clinical diagnoses (e.g. loss of consciousness, superficial soft tissue injuries) or surgically identified injuries of the extremities.

The ISS of patients without missed injuries was compared with the ISS of patients with missed non-relevant injuries, and the differences were not significant $(P=0.818)$. In contrast, the mean ISS in patients with relevant missed injuries was significantly higher than among patients who had no missed injuries $(P=0.003)$ (Table 5). 
Table 5 Mean ISS values and standard deviations (SD) for the different groups. Differences were tested for (a) missed injuries with no relevance compared with no missed injuries and (b) missed injuries with relevance compared with no missed injuries. ISS was significantly higher in patients with missed findings of clinical relevance

\begin{tabular}{lrrll}
\hline Type of MI & $\boldsymbol{n}$ & \multicolumn{1}{c}{$\%$} & Mean ISS (SD) & $\boldsymbol{P}^{*}$ \\
\hline None & 336 & 89.6 & $25.8( \pm 17.0)$ & - \\
With no relevance & 14 & 3.7 & $23.6( \pm 14.0)$ & 0.818 \\
With relevance & 25 & 6.7 & $36.1( \pm 15.5)$ & 0.003 \\
All patients & 375 & 100 & $26.6( \pm 17.0)$ & \\
\hline
\end{tabular}

*Compared with no missed injuries group

ISS, Injury Severity Score; MI, missed injuries

The re-evaluation by the radiologist who was aware of the missed lesion showed that 41 of the 48 injuries $(85.4 \%)$ that were initially missed could have been retrospectively detected on the CT images. The injuries that were not visible on secondary reading because of methodical limitations were: tears of the colonic serosa $(n=3)$, and contusions of the colon $(n=2)$, small bowel $(n=1)$, and pancreas $(n=1)$. All seven findings were proven and documented in the reports from laparotomy; consequently, those had a clinical impact.

One fracture of the sacrum was seen on the initial CT, and was not documented in the discharge report.

\section{Discussion}

Missed injuries in diagnostic imaging of trauma patients are inevitable, and are more commonly seen in patients with multiple injuries. In our study, injuries were missed in 39 of 375 patients $(10.4 \%)$; 25 of 375 patients $(6.7 \%)$ had missed injuries that had clinical impact and were further treated.

Compared with other studies, the rate of missed injuries that had further clinical relevance in our study was in the lower third of the reported range of $1.3 \%$ to $47 \%(13-22)$. As definitions of "missed injury" and the imaging methods show relevant variations, incidence rates are difficult to compare between those studies.

Additionally, the percentage of correct CT diagnoses of the final written report was relatively high (about 90\%) in our study compared with published data (21). This could be explained by the fact that the final written report included the potential corrections made by an experienced attending radiologist during the clinical second reading process. This approach differs substantially from methods in other studies. However, we sought to identify lesions which are prone to be missed despite the interpretation by clinically experienced readers in contrast to the detection of injuries which could be missed by the first reader. Furthermore, we defined the combination of all available clinical follow-up data as reference standard instead of cross-checking by an experienced reader only (21). Consequently, we were able to distinguish between lesions whose misdiagnosis might be avoidable and those which could be considered as truly not detectable due to inherent limitations of CT.
Most of the missed injuries were bone fractures, which corresponds well with the results from other studies $(13-20,23,24)$. In patients who sustained severe trauma with multiple injuries, the incidence of fractures is obviously high. Missed fractures of single ribs $(n=5)$, transverse processes of vertebrae $(n=4)$, and nasal bones $(n=2)$, contributed most to the group with injuries without further relevance (Table 3). These injuries seemed to be missed often because the patients had co-existing injuries that were more serious or life-threatening.

In the group of missed injuries that required further treatment or were indicators of a severe trauma, fractures were also the most common type of injury $(n=9)$. In contrast to the first group, these fractures had further impact on treatment and were consequently rated as relevant. Moreover, one subtle epidural hematoma was missed in the initial CT, which had no additional therapeutic consequence because the patient had intracranial hemorrhage in the contralateral hemisphere that required craniotomy anyway (Fig. 1). There was also one missed diffuse axonal injury (DAI) in a patient who also had suffered an intracranial hemorrhage. Fig. 2 shows a case of duodenal rupture that was initially missed but was detected with secondary reading of the images.

According to the study design, the radiologist who performed the second reading was aware what kinds of lesions were missed in the written report. We chose this method for focusing on analyzing limitations of the imaging methods or equipment instead of testing the performance of the reading team. The fact that most of the initially missed lesions were detected in the original CT data by the radiologist experienced in emergency radiology indicates that the CT equipment used for this study as well as the imaging methods were adequate to diagnose trauma-related injuries. We did not investigate if the use of an advanced CT scanner with more than four-detector rows would have declined the rate of missed findings. Whereas an increased number of detector rows influences the spatial resolution in the $\mathrm{z}$ direction, the $\mathrm{xy}$-plane resolution within axial slices and the resulting sensitivity for traumatic injuries can be considered as comparable. In conclusion, the use of a CT system with more than four-detector rows would have the same sensitivity in this particular setting (secondary, retrospective reading for known injuries). In terms of diagnostic confidence, however, an additional, prospective value can be expected due to improved coronal and sagittal images.

Even with secondary reading, approximately $7 / 48$ of the injuries primarily missed were still not detected. Most of these cases were superficial injuries to the serosa of colon or small bowel without laceration or rupture of the bowel wall. All lesions were reported in patients who had laparotomy for other reasons. Ultimately, in all of these cases, it was unclear whether the missed lesions would have had any impact had they been left untreated. Nevertheless, the injuries had to be rated as relevant according to our definition. Bowel injuries are often missed in CT examinations, particularly when they are subtle (25). Studies have reported a sensitivity for detection of bowel injuries with CT of $69-95 \%$ with a specificity of $94-100 \%$, depending of the 

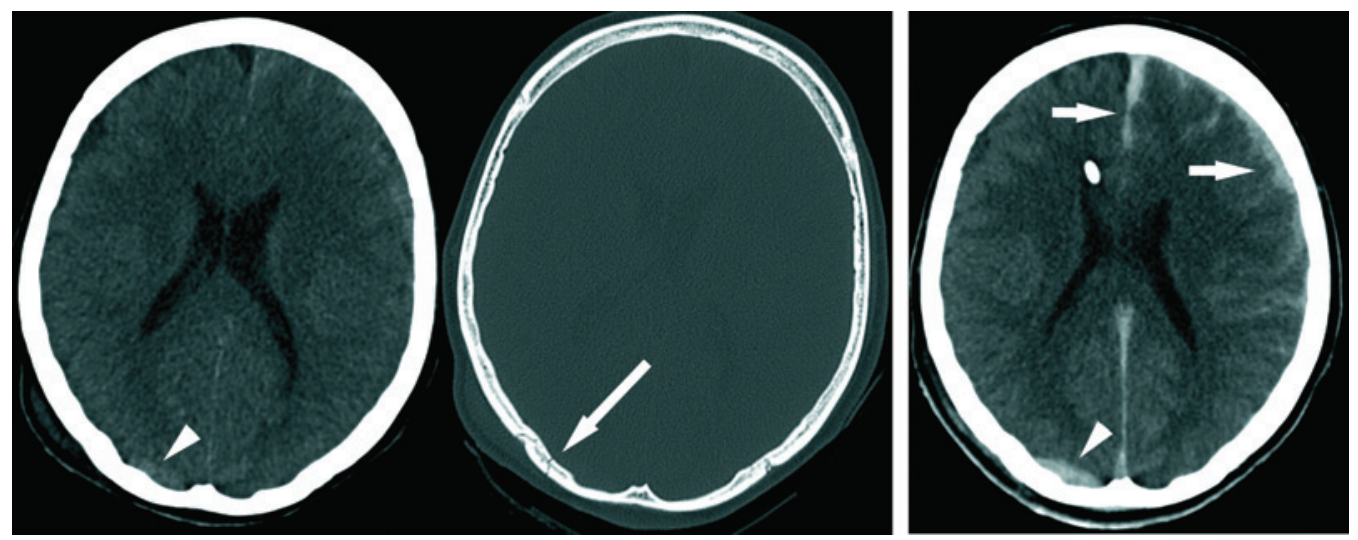

Fig. 1 (a) Example of a missed injury that was thought not to have any clinical impact because the treatment was not changed. Transverse sections of a non-enhanced head CT from a 42-year-old woman, who had blunt injuries from a motor vehicle collision. On the left image, a small epidural hematoma (arrowhead) is visible over the right occipital lobe, which was not mentioned in the written report although the adjacent fracture (arrow on the right section) was documented. There was also a subarachnoid and a subdural hemorrhage over the left hemisphere. (b) Image from the same patient obtained 73 min after the initial CT. The progression of the epidural (arrowhead) as well as the subdural and subarachnoid (arrows) hemorrhages was reported at this examination. In the time between the examinations, the patient had external ventriculostomy to the right frontal horn of the right side ventricle. Because of increasing intracranial pressure, the patient then had left-sided trepanation

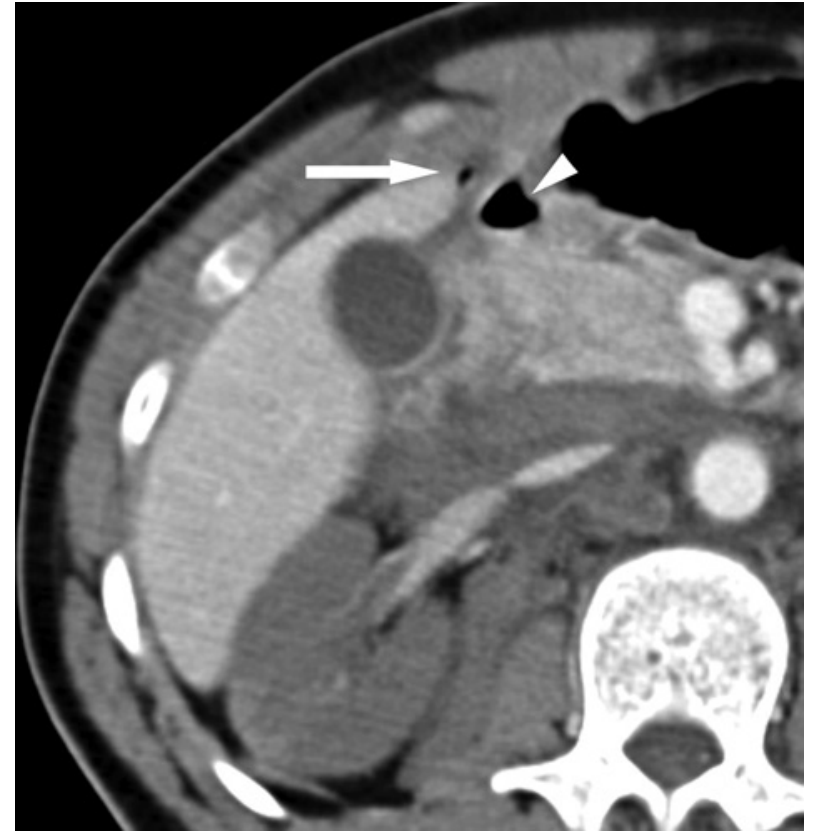

Fig. 2 Transverse contrast-enhanced CT section of the right upper abdominal quadrant of a 28-year-old construction worker who was hit by a pack of wooden boards falling from a height. The patient had a grade IV liver laceration, an injury to the right renal artery causing kidney infarction, and a retroperitoneal hematoma. The small bubble of free air (arrow) adjacent to the duodenum (arrowhead) was missed. The patient had a laparotomy for the injuries to the liver and renal artery, which also showed a rupture of the descending part of the duodenum

imaging protocols and CT scanners used (26, 27). Oral contrast medium is sometimes used to distend the bowel loops but is not necessarily needed to improve the diagnostic performance (28). In the missed findings of this study, oral contrast could have reduced the number of missed lesions. Most of the patients in this study cohort were severely injured with a mean ISS of 26 , and oral contrast is not given routinely to such patients in our institution because it is time-consuming. There were also superficial bleedings from pancreatic contusion that were not seen at the secondary reading (Fig. 3). In contrast to other studies, we did not observe missed injuries that had serious consequences for the patients, such as unstable spinal fractures. However, we cannot exclude further subtle, missed injuries, which were not seen with $\mathrm{CT}$ and were clinically not apparent.

Relevant injuries were more common in patients with higher ISS (Table 5). This also corresponds well with results of other studies (15, 18, 20). With increasing numbers of injuries, the risk of missing injuries also increases. In light of this background, these patients should be re-evaluated carefully in a clinical setting to avoid delayed diagnoses.

In contrast to other studies, we analyzed the incidence of missed injuries of the initial CT examination and did not take follow-up scans into account. Consequently, lesions were rated as "missed" if they were not included in the initial CT report, even when they were detected by surgery or follow-up imaging studies. This criterion is different from those in most other studies that dealt with delayed or missed diagnoses. Some of those focused mainly on missed lesions in clinical departments, such as surgery and emergency medicine rather than radiology $(15,17,18)$, did not use WBCT $(17,20)$, or did not provide their specific imaging protocols $(14,16)$. In most of these studies, injuries were classified as missed if they were not recognized by any means within $24 \mathrm{~h}$, including follow-up CT imaging. However, we justify our study methods as we consider the first CT scan as most important to avoid delayed diagnoses.

In addition, external factors which could have caused missed or delayed findings deserve discussion. Bardenheuer et al. (29) have shown that admissions of trauma patients to the hospital occur remarkably more often during offhours compared to regular working hours. It is also known that different call types (e.g. overnight, weekend, daytime) cause discrepancies between residents' preliminary interpretations and attending radiologists' final 


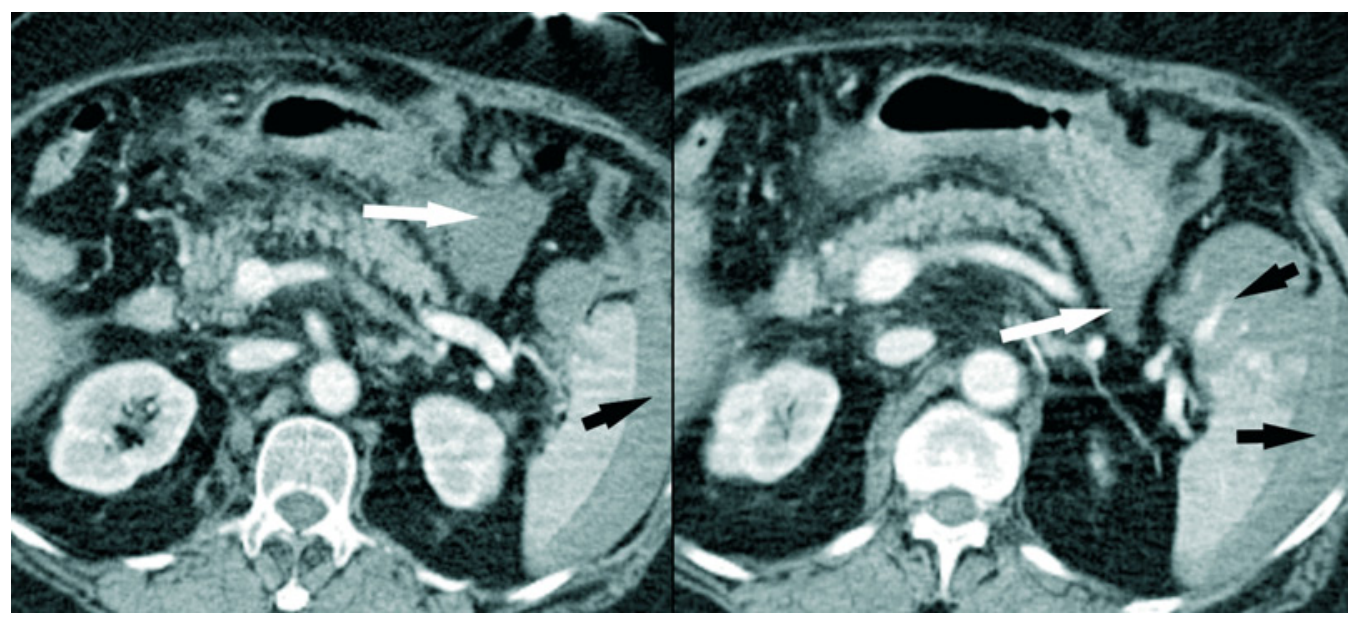

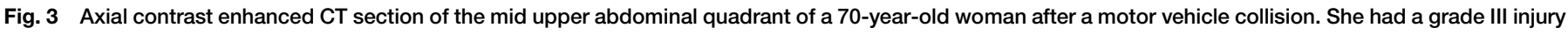
to the spleen (black arrows), a rupture of some peripheral mesenteric arteries, and a rupture of the ileum. Fluid collections were reported in the omental bursa (white arrows) and in other parts of the abdomen not shown in these images. The patient then had a laparotomy because of the intraperitoneal hemorrhage. During the inspection of the intraperitoneal cavity a contusion of the pancreas with two small bleeding areas was seen and sutured. These findings were not reported on the initial CT. Even in retrospect, the pancreatic lesions were not detected and therefore defined as missed injury

interpretations (30). The fact that during early hours the diagnostic capabilities are assumingly suboptimal might partially contribute to this observation. However, the absolute discrepancy rate is considered as minimal and without detrimental effects on patient care $(31,32)$. At our institution, the radiologist on call has a 20-h shift on weekdays (12:00 to $08: 00)$ and $24-\mathrm{h}$ shift on weekends (08:00 to 08:00). Therefore, our results should be consistent within this study population. Nevertheless, a second reading of each trauma CT is established at our institution. This process of quality assurance is performed as soon as possible in a clinical setting: during regular working hours (08:00 to 17:00), the attending radiologist is present at the department and cross-checks the initial report immediately after the CT scan. During off-hours, all images and CT reports were reviewed at the beginning of the following working day.

The major limitation of this study is its retrospective design. It was not possible to exclude that some of the missed lesions had been detected and communicated orally to the trauma surgeons, but were not mentioned in the written report. Second, we could not calculate exactly the extent of delay in the final diagnosis of the missed lesions and its influence on patient's outcome. However, we wish to emphasize that the purpose of our study was to identify those injuries which are prone to be missed by interpretation of the initial WBCT after multiple trauma. We aimed to identify those lesions whose misdiagnosis would be avoidable if the radiologist was aware of them. As this is a scarce topic in literature and MDCT is the current first line imaging tool in patients with multiple injuries, these results are clinically relevant. Nevertheless, future studies are needed to evaluate the clinical impact of increased awareness of commonly missed injuries. Third, we did not analyze the influence on the final written report by clinical communication with the surgeons and critical care colleagues. As the interdisciplinary approach is one of the most critical fundaments of our institutional trauma algorithm, there is a continuous sharing of information during the initial trauma work-up which cannot be evaluated as a separate parameter. However, as the interdisciplinary concept is supported and recommended by clinical guidelines, it can be considered as representative for evidence-based medicine. Fourth, we are not able to exclude that further lesions could have been missed, in particular, if they remained clinically asymptomatic. However, we combined all available clinical data to minimize this error. In addition, patients who died immediately were not analyzed according to our exclusion criteria. Nevertheless, 14 patients died during the subsequent phase after trauma. Unfortunately, the access on autopsy reports was very restricted and therefore these data were not taken into account. However, recent studies intend to establish CT as supplemental modality to autopsy for postmortem assessment, so-called virtopsy (33). It remains to be seen to what extent these results might contribute to this topic. Furthermore, we did not evaluate the incidence of missed fractures of the extremities. Including these injuries, the rate of delayed diagnoses is assumed to be higher than reported in this study. Since extremity fractures are usually diagnosed by plain radiography, we excluded these injuries from analysis.

In conclusion, the presented data allow for the first time to evaluate WBCT in multiple trauma for its limitations in detecting all clinically relevant injuries. We have shown that even in dedicated trauma centers some of the trauma-related diagnoses are missed in the primary CT image reading. However, only few of these missed injuries are clinically relevant. This indicates the need for proper training of residents on call as well as thorough crosschecking by attending radiologists alert to possibly missed findings. In terms of quality assurance, a systematic radiological as well as interdisciplinary re-evaluation of diagnostic results is recommended to increase awareness of common and subtle findings with the intention of avoiding delayed diagnosis.

\section{Conflict of interest: None.}




\section{REFERENCES}

1 Geijer M, El-Khoury GY. MDCT in the evaluation of skeletal trauma: principles, protocols, and clinical applications. Emerg Radiol 2006;13:7-18

2 Huber-Wagner S, Lefering R, Qvick LM, et al. Effect of whole-body CT during trauma resuscitation on survival: a retrospective, multicentre study. Lancet 2009;373:1455-61

3 Marmery H, Shanmuganathan K. Multidetector-row computed tomography imaging of splenic trauma. Semin Ultrasound CT MR 2006;27:404-19

4 Mirvis SE, Shanmuganagthan K. Imaging hemidiaphragmatic injury. Eur Radiol 2007;17:1411-21

5 Mirvis SE, Shanmuganathan K. Diagnosis of blunt traumatic aortic injury 2007: still a nemesis. Eur J Radiol 2007;64:27-40

6 Poletti PA, Mirvis SE, Shanmuganathan K, et al. Blunt abdominal trauma patients: can organ injury be excluded without performing computed tomography? J Trauma 2004;57:1072-81

7 Salim A, Sangthong B, Martin M, et al. Whole body imaging in blunt multisystem trauma patients without obvious signs of injury: results of a prospective study. Arch Surg 2006;141:468-73

8 Self ML, Blake AM, Whitley M, et al. The benefit of routine thoracic, abdominal, and pelvic computed tomography to evaluate trauma patients with closed head injuries. Am J Surg 2003;186:609-13

9 Shanmuganathan K, Mirvis SE, Chiu WC, et al. Penetrating torso trauma: triple-contrast helical CT in peritoneal violation and organ injurya prospective study in 200 patients. Radiology 2004;231:775-84

10 Sliker CW, Mirvis SE. Imaging of blunt cerebrovascular injuries. Eur J Radiol 2007;64:3-14

11 Traub M, Stevenson M, McEvoy S, et al. The use of chest computed tomography versus chest $\mathrm{X}$-ray in patients with major blunt trauma. Injury 2007;38:43-7

12 Dreizin D, Munera F. Blunt polytrauma: evaluation with 64-section whole-body CT angiography. Radiographics 2012;32:609-31

13 Biffl WL, Harrington DT, Cioffi WG. Implementation of a tertiary trauma survey decreases missed injuries. J Trauma 2003;54:38-43

14 Brooks A, Holroyd B, Riley B. Missed injury in major trauma patients. Injury 2004;35:407-10

15 Buduhan G, McRitchie DI. Missed injuries in patients with multiple trauma. J Trauma 2000;49:600-5

16 Gales H, Perry M. Is there a role for planned serial chest radiographs and abdominal ultrasound scans in the resuscitation room following trauma? Ann R Coll Surg Engl 2006;88:535-9

17 Hoff WS, Sicoutris CP, Lee SY, et al. Formalized radiology rounds: the final component of the tertiary survey. J Trauma 2004;56:291-5
18 Houshian S, Larsen MS, Holm C. Missed injuries in a level I trauma center. J Trauma 2002;52:715-9

19 Okello CR, Ezati IA, Gakwaya AM. Missed injuries: a Ugandan experience. Injury 2007;38:112-7

20 Pehle B, Kuehne CA, Block J, et al. The significance of delayed diagnosis of lesions in multiply traumatised patients. A study of 1,187 shock room patients. Unfallchirurg 2006;109:964-74

21 Eurin M, Haddad N, Zappa M, et al. Incidence and predictors of missed injuries in trauma patients in the initial hot report of whole-body CT scan. Injury 2012;43:73-7

22 Pfeifer R, Pape HC. Missed injuries in trauma patients: A literature review. Patient Saf Surg 2008;2:20

23 Platzer P, Hauswirth N, Jaindl M, et al. Delayed or missed diagnosis of cervical spine injuries. J Trauma 2006;61:150-5

24 Tadros AM, Lunsjo K, Czechowski J, et al. Causes of delayed diagnosis of scapular fractures. Injury 2008;39:314-8

25 Stuhlfaut JW, Anderson SW, Soto JA. Blunt abdominal trauma: current imaging techniques and CT findings in patients with solid organ, bowel, and mesenteric injury. Semin Ultrasound CT MR 2007;28:115-29

26 Brofman N, Atri M, Hanson JM, et al. Evaluation of bowel and mesenteric blunt trauma with multidetector CT. Radiographics 2006;26:1119-31

27 Jacobs JE, Megibow AJ. CT of GI trauma. Crit Rev Comput Tomogr 2004:45:157-80

28 Stuhlfaut JW, Soto JA, Lucey BC, et al. Blunt abdominal trauma: performance of $\mathrm{CT}$ without oral contrast material. Radiology 2004;233:689-94

29 Bardenheuer M, Obertacke U, Waydhas C, et al. Epidemiology of the severely injured patient. A prospective assessment of preclinical and clinical management. AG Polytrauma of DGU. Unfallchirurg 2000;103:355-63

30 Davenport MS, Ellis JH, Khalatbari SH, et al. Effect of work hours, caseload, shift type, and experience on resident call performance. Acad Radiol 2010;17:921-7

31 Chung JH, Strigel RM, Chew AR, et al. Overnight resident interpretation of torso CT at a level 1 trauma center an analysis and review of the literature. Acad Radiol 2009;16:1155-60

32 Ruchman RB, Jaeger J, Wiggins EF 3rd, et al. Preliminary radiology resident interpretations versus final attending radiologist interpretations and the impact on patient care in a community hospital. Am J Roentgenol 2007;189:523-6

33 Bolliger SA, Thali MJ, Ross S, et al. Virtual autopsy using imaging: bridging radiologic and forensic sciences. A review of the Virtopsy and similar projects. Eur Radiol 2008;18:273-82 